(1974) for hydrogen bonds involving ureido groups. The latter investigators point out that the ureido groups are hydrogen-bond acids and that they therefore form long hydrogen bonds when used as acceptors. The second amino hydrogen $\mathrm{H}(\mathrm{N} 4 A)$ does not form any hydrogen bonds. Cases where a potential hydrogen donor is not involved in any close contacts are very rare. $\mathrm{H}(\mathrm{N} 4 A)$ appears to be directed towards the center of the phenyl ring of the molecule related by the transformation $\frac{1}{2}-x,-\frac{1}{2}+$ $y, \frac{3}{2}-z$. The closest atoms are $\mathrm{C}(5)$ and $\mathrm{C}(6)$ : $\mathrm{N}(4) \cdots \mathrm{C}(5) \quad 3.508(2), \quad \mathrm{H}(\mathrm{N} 4 A) \cdots \mathrm{C}(5) \quad 2.66(2)$; $\mathrm{N}(4) \cdots \mathrm{C}(6) \quad 3.480(2), \mathrm{H}(\mathrm{N} 4 A) \cdots \mathrm{C}(6) \quad 2.69$ (2) $\AA$. If this interaction between the amino group and the $\pi$ cloud of the phenyl ring is energetically favorable, it may also contribute to the weakening of the hydrogen bond involving $\mathrm{H}(\mathrm{N} 4 B)$. The only other short intermolecular contact is the 3.240 (2) $\AA$ contact between $\mathrm{C}(5)$ and $\mathrm{O}(10)\left(\frac{1}{2}-x, \frac{1}{2}+y, \frac{1}{2}-z\right)$.

Research supported in part by the Ciba Geigy Corporation and by grant RR-05716 from the National Institutes of Health, DHHS.

\section{References}

Banting, L., Smith, H. J., James, M., Jones, G., NaZareth, W., Nicholls, P. J., Hewlins, M. J. E. \& Rowlands, M. G. (1988). J. Enzyme Inhib. 2, 215-229.

Blessing, R. H. (1987). Crystallogr. Rev. 1, 3-57.

Camacho, A. M., Brough, A. J., Cash, R. \& Wilroy, R. (1966). J. Pediatr. 68, 852-853.

Enraf-Nonius (1979). Structure Determination Package. EnrafNonius, Delft, The Netherlands.

Finch, N., Dziemian, R., Cohen, J. \& Steinetz, B. G. (1975). Experientia, 31, 1002-1003.

Gartland, G. L. \& Craven, B. M. (1974). Acta Cryst. B30, 980-987.

Germain, G., Main, P. \& Woolfson, M. M. (1971). Acta Cryst. A27, 368-376.

JoHNSON, C. K. (1976). ORTEPII. Report ORNL-5138. Oak Ridge National Laboratory, Tennessee, USA.

KIM, S.-H. \& Rich, A. (1968). Proc. Natl Acad. Sci. USA, 60, 402-408.

Santen, R. J., Worgul, T. J., Samojlik, E., Boucher, A. E., Lipton, A. \& HaRvey, H. (1982). Cancer Res. Suppl. 42, 3397s.

Shaw, M. A., Nicholls, P. J. \& Smith, H. J. (1988). J. Steroid Biochem. 31, 137-146.

Stout, G. H. \& JenSEN, L. H. (1968). X-ray Structure Determination. New York: Macmillan.

VOET, D. (1972). J. Am. Chem. Soc. 94, 8213-8222.

Williams, P. P. (1973). Acta Cryst. B29, 1572-1579.

Williams, P. P. (1974). Acta Cryst. B30, 12-17.

Acta Cryst. (1991). C47, 832-835

\title{
Structure of the Modified Nucleoside $2^{\prime}, 3^{\prime}$-Dideoxy-3'-fluorocytidine*
}

\author{
By H. L. De Winter, N. M. Blaton, O. M. Peeters and C. J. De Ranter $\dagger$ \\ Laboratorium voor Analytische Chemie en Medicinale Fysicochemie, \\ Instituut voor Farmaceutische Wetenschappen, Katholieke Universiteit Leuven, Van Evenstraat 4, \\ B-3000 Leuven, Belgium
}

And A. Van Aerschot And P. Herdewijn

Laboratorium voor Farmaceutische Chemie, Rega Institute for Medical Research, Katholieke Universiteit Leuven, B-3000 Leuven, Belgium

(Received 20 April 1990; accepted 7 August 1990)

\begin{abstract}
Dideoxy-3-fluoro- $\beta$-D-erythro-pentofuranosyl)cytosine, $\mathrm{C}_{9} \mathrm{H}_{12} \mathrm{FN}_{3} \mathrm{O}_{3}, M_{r}=229 \cdot 21$, triclinic, $\quad P 1, \quad a=6.997(4), \quad b=7.396(4), \quad c=$ 10.639 (5) $\AA, \quad \alpha=94.48(4), \quad \beta=107.74(4), \quad \gamma=$ $104.40(4)^{\circ}, V=500 \cdot 8(5) \AA^{3}, Z=2, D_{m}=1 \cdot 52, D_{x}=$ $1.520 \mathrm{Mg} \mathrm{m}^{-3}, \quad \lambda(\mathrm{Mo} K \alpha)=0.71069 \AA, \quad \mu=$ $0.1198 \mathrm{~mm}^{-1}, F(000)=240, T=293 \mathrm{~K}$, final $R=$ 0.033 for 2321 unique observed $[F \geq 4 \sigma(F)]$ reflections. The asymmetric unit contains two molecules $A$

\footnotetext{
* Structural Studies of Modified Nucleosides. Part VI. Part V: Everaert, Peeters, Blaton, De Ranter, Van Aerschot \& Herdewijn (1991).

$\dagger$ To whom correspondence should be addressed.
}

0108-2701/91/040832-04\$03.00 and $B$. For molecule $A$, the $N$-glycosidic torsion angle $\chi$ has a value of $-143.5(3)^{\circ}$, the sugar pucker is mixed ${ }^{2} T_{1}{ }^{2} E$ with $P=154$ (1) $\left(\mathrm{C}^{\prime}\right.$ endo) and $\psi_{m}$ $=40(1)^{\circ}$, and the $\mathrm{O}^{\prime} A-\mathrm{C}^{\prime} A-\mathrm{C}^{\prime} A-\mathrm{C}^{\prime} A$ torsion angle $\gamma=63.4(4)^{\circ}$. For molecule $B, \chi=$ $-153.0(3), \gamma=-71.4(4)^{\circ}$ and the sugar pucker is ${ }^{2} E$ with $P=164(1)\left(\mathrm{C} 2^{\prime}\right.$ endo $)$ and $\psi_{m}=36(1)^{\circ}$. The packing of the crystal is determined by a network of hydrogen bonds. Base pairing between $A$ and $B$ occurs, and in this way a pseudo-inversion centre is formed between the two bases. The conformational parameters are in accordance with the IUPAC-IUB Joint Commission on Biochemical Nomenclature [Pure Appl. Chem. (1983), 55, 1273-1280] guidelines.

(C) 1991 International Union of Crystallography 
Experimental. The crystal structure of the title compound has been determined as part of a continuing program of investigation of potentially antiviral modified nucleosides, with particular reference to possible anti-AIDS compounds. The method of preparation of the product has been described by Herdewijn, Balzarini, De Clercq, Pauwels, Baba, Broder \& Vanderhaeghe (1987). Colourless prismatic crystals from a methanol-amyl acetate solution, $0 \cdot 3$ $\times 0.4 \times 0.5 \mathrm{~mm}$. Density measured by flotation in $n$-heptane $/ \mathrm{CCl}_{4}$. Weissenberg photographs show no systematically absent reflections. Stoe STADI-4 diffractometer, cell constants by least-squares refinement of the setting angles of 24 reflections with $20 \leq 2 \theta \leq 30, \omega / 2 \theta \operatorname{scan},[(\sin \theta) / \lambda]_{\max }=0.7035 \AA^{-1}$, $0 \leq h \leq 10,-10 \leq k \leq 10,-15 \leq l \leq 15$. Intensities of three standard reflections $(200,020,002)$ monitored every hour showed no significant decrease in intensity, 3143 reflections measured, 2920 unique reflections of which 2321 were considered observed with $F \geq 4 \sigma(F)$. Data reduction with REDU4 (Stoe \& Co., 1985), Lorentz and polarization corrections, no absorption corrections $\left(\mu=0.1198 \mathrm{~mm}^{-1}\right)$. Scattering factors were taken from International Tables for X-ray Crystallography (1974, Vol. IV, Table 2.2B) and for $\mathrm{H}$ atoms from Stewart, Davidson \& Simpson (1965). Anomalous-dispersion corrections were included for all non-H atoms (Ibers \& Hamilton, 1964). Initial attempts to solve the structure with MULTAN82 (Main, Fiske, Hull, Lessinger, Germain, Declercq \& Woolfson, 1982) resulted always in the well known 'chicken-wire' $E$ maps. Modification of the default input parameters did not resolve this problem. Comparison of the averaged powers of the normalized structure factors $E$ with comparable theoretical values suggested a pseudo-centrosymmetric symmetry for the crystal. Therefore, as an alternative approach to solving the phase problem, the vector-search method was tried. In space group $P 1$, only the orientation of the molecules with respect to the crystallographic axes has to be determined. For this purpose, the 1-methylcytosine skeleton was used as an input model for the vector-search rotationfunction program ORIENT (Beurskens, Beurskens, Strumpel \& Nordman, 1987). A default run, with an initial average step scan of 10 and a $0.3 \AA$ grid for the Patterson map did not reveal the correct orientation of the fragment. A second run, with an initial average step scan of $5^{\circ}$, was more successful, and the correctly oriented fragment was subsequently used as input for DIRDIF (Beurskens, Bosman, Doesburg, Van den Hark, Prick, Noordik, Beurskens, Gould \& Parthasarathi, 1983), which revealed 26 of the 32 non- $\mathrm{H}$ atoms. The remaining atoms were located in a subsequent difference map. Refinement on $F$ by fullmatrix least squares, first with isotropic temperature factors and finally anisotropically. All $\mathrm{H}$ atoms were found in a difference synthesis and they were included in the refinement with a fixed temperature factor $B=4.0 \AA^{2}$. Final $R=0.033, w R=0.041$, with $w=1 /\left[\sigma^{2}\left(F_{o}\right)+0.0004 F_{o}^{2}\right], S=1.43$. Largest parameter shift/e.s.d $=0.02$. Minimum and maximum residual electron density -0.21 and $0.20 \mathrm{e} \AA^{-3}$. The number of reflections per refined parameter 2321/358 $=6.5$. All calculations were performed on a Digital PDP-11/73 and MicroVAX 2000 microcomputer using SDP (Enraf-Nonius, 1985) and PARST (Nardelli, 1983).

Discussion. A PLUTO view (Motherwell \& Clegg, 1978) of the title compound with the atomic numbering scheme is shown in Fig. 1.* The final atomic coordinates and equivalent isotropic thermal parameters are given in Table 1. Bond lengths, bond angles and selected torsion angles are given in Table 2. Table 3 gives the geometry of all hydrogen bonds.

A least-squares fit procedure with the program BMFIT (Nyburg, 1974) on the atoms of the cytosine base showed a close geometrical similarity between the cytosine bases of $A$ and $B$ (r.m.s. deviation = $0.039 \AA$ ). Except for the $\mathrm{C} 2-\mathrm{O} 2$ bond length of molecule $A[1.253(3) \AA]$, which is longer than the standard $\mathrm{C}=\mathrm{O}$ distance of $1 \cdot 215 \AA$, all other bond

\begin{abstract}
* Lists of structure factors, anisotropic thermal parameters, bond lengths and angles involving $\mathbf{H}$ atoms, least-squares planes and $\mathrm{H}$-atom parameters have been deposited with the British Library Document Supply Centre as Supplementary Publication No. SUP 53460 (29 pp.). Copies may be obtained through The Technical Editor, International Union of Crystallography, 5 Abbey Square, Chester CH1 2HU, England.
\end{abstract}

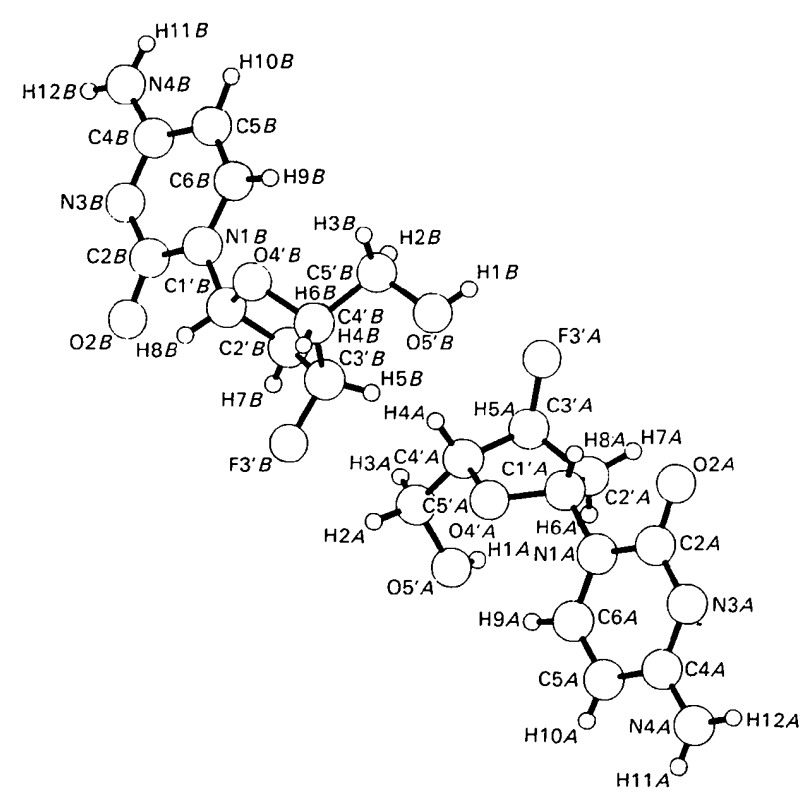

Fig. 1. PLUTO plot (Motherwell \& Clegg, 1978) of the title compound with atomic numbering scheme. 
Table 1. Atomic coordinates and equivalent isotropic temperature factors $\left(\AA^{2} \times 10^{4}\right)$ with e.s.d.'s in parentheses

\begin{tabular}{|c|c|c|c|c|}
\hline & $x$ & $y$ & $z$ & $U_{\mathrm{cq}}$ \\
\hline $\mathrm{N} 1 A$ & $-0.0907(2)$ & $0.6872(2)$ & $0.0697(2)$ & 304 (4) \\
\hline $\mathrm{C} 2 \mathrm{~A}$ & $-0.1364(3)$ & $0.8329(3)$ & $0.0009(2)$ & $306(5)$ \\
\hline $\mathrm{O} 2 A$ & $-0.2949(2)$ & $0.8824(2)$ & $0.0014(2)$ & 449 (4) \\
\hline $\mathrm{N} 3 A$ & -0.0068 & $0 \cdot 9188(3)$ & $-0.0622(2)$ & $330(4)$ \\
\hline $\mathrm{C} 4 \mathrm{~A}$ & 0.1642 & 0.8657 (3) & $-0.0568(2)$ & $283(5)$ \\
\hline $\mathrm{N} 4 A$ & $0.2897(3)$ & $0.9594(3)$ & $-0.1180(2)$ & 360 (4) \\
\hline $\mathrm{C} 5 \mathrm{~A}$ & $0.2115(3)$ & $0.7147(3)$ & 0.0099 (2) & $346(5)$ \\
\hline $\mathrm{C} 6 A$ & $0.0806(3)$ & $0.6277(3)$ & $0.0705(2)$ & $341(5)$ \\
\hline $\mathrm{Cl}^{\prime} A$ & $-0.2368(3)$ & $0.6916(3)$ & $0.1334(2)$ & $307(5)$ \\
\hline $\mathrm{C} 2^{\prime} A$ & $-0.3856(3)$ & $0.4048(3)$ & $0.0534(2)$ & 379 (5) \\
\hline $\mathrm{C}^{\prime} A$ & $-0.4499(3)$ & $0.2130(3)$ & $0 \cdot 1609(2)$ & $357(5)$ \\
\hline $\mathrm{F} 3^{\prime} A^{*}$ & -0.602 & 0.392 & 0.185 & $355(4)$ \\
\hline$C 4^{\prime} A$ & $-0.2522(3)$ & 0.3796 (3) & $0.2829(2)$ & $303(5)$ \\
\hline $\mathrm{O} 4^{\prime} A$ & $-0.1211(2)$ & 0.5464 (2) & $0.2563(2)$ & $345(4)$ \\
\hline $\mathrm{CS}^{\prime} \mathrm{A}$ & $-0.1318(4)$ & $0.2354(3)$ & $0.3153(3)$ & $406(5)$ \\
\hline $\mathrm{OS}^{\prime} A$ & $-0.0538(3)$ & $0.1866(3)$ & $0.2129(2)$ & $545(5)$ \\
\hline$N \mid B$ & $-0.7225(2)$ & $-0.4424(2)$ & 06593 (2) & $286(4)$ \\
\hline$C 2 B$ & $-0.6810(3)$ & $-0.6004(3)$ & $0.7154(2)$ & $278(4)$ \\
\hline $\mathrm{O} 2 B$ & $-0.5138(2)$ & $-0.6331(2)$ & $0.7223(2)$ & $412(4)$ \\
\hline $\mathrm{N} 3 B$ & $-0.8257(2)$ & $-0.7096(2)$ & $0.7599(2)$ & $300(4)$ \\
\hline$C 4 B$ & $-1.0079(3)$ & $-0.6712(3)$ & $0.7444(2)$ & 269 (4) \\
\hline $\mathrm{N} 4 B$ & $-1 \cdot 1416(3)$ & $-0.7760(3)$ & $0.7951(2)$ & $363(5)$ \\
\hline $\operatorname{Cs} B$ & $-1.0602(3)$ & $-0.5221(3)$ & $0.6764(2)$ & $332(5)$ \\
\hline C $6 B$ & $-0.9127(3)$ & $-0.4101(3)$ & $0.6374(2)$ & $316(5)$ \\
\hline $\mathrm{Cl}^{\prime} B$ & $-0.5594(3)$ & -0.3229 & $0.6160(2)$ & $307(5)$ \\
\hline$C 2^{\prime} B$ & $-0.5812(3)$ & -0.3859 (3) & $0.4717(2)$ & $385(5)$ \\
\hline$C^{\prime} B$ & $-0.4811(3)$ & $-0.2022(3)$ & $0.4341(2)$ & $409(5)$ \\
\hline $\mathrm{F}^{\prime} B$ & $-0.2621(2)$ & $-0.1686(3)$ & $0.4801(2)$ & 704 (5) \\
\hline$C 4^{\prime} B$ & $-0.5387(3)$ & $-0.0558(3)$ & $0.5112(2)$ & $341(5)$ \\
\hline$O 4^{\prime} B$ & $-0.5806(3)$ & $-0.1371(2)$ & $0.6230(2)$ & $373(4)$ \\
\hline$C^{\prime} B$ & $-0.7321(3)$ & -0.0070 & $0.4296(2)$ & $421(5)$ \\
\hline $\mathrm{OS}^{\prime} B$ & $-0.6731(3)$ & $0.0992(3)$ & $0.3350(2)$ & $663(5)$ \\
\hline
\end{tabular}

* Positional parameters kept fixed during the refinement.

lengths and bond angles are normal (for tables, see Allen, Kennard, Watson, Brammer, Orpen \& Taylor, 1987). The O2's of modified cytidine nucleosides with a similar elongated $\mathrm{C} 2-\mathrm{O} 2$ bond length are always involved in strong hydrogen bonding (Lalitha, Ramakumar \& Viswamitra, 1989).

The pyrimidine heterocycles of both bases are almost planar, with only minor deviations from the weighted least-squares planes [max. deviation for molecule $A$ : $-0.014(3) \AA \mathrm{C} 4 A$; for molecule $B$ : 0.033 (3) $\AA \mathrm{C} 2 B]$.

Since the geometry of both bases is almost identical, a least-squares fit [BMFIT; Nyburgh (1974)] on the atoms of the bases reveals some conformational differences in the sugar rings and their substituents: an r.m.s. deviation of $0.742 \AA$ between the atoms of the sugar rings and substituents (atoms $\mathrm{Cl}^{\prime}$ through $\mathrm{O5}^{\prime}$ ) was calculated. A minor deviation is found in the orientation of the base relative to the sugar moiety, which globally is anti for both molecules, but $\chi=-143.5$ (3) for $A$ and $-153.0(3)^{\circ}$ for $B$. The ${ }^{2} T_{1} /{ }^{2} E$ and ${ }^{2} E$ puckers of $A$ and $B$, respectively, together with the puckering amplitudes of 40 (1) and $36(1)^{\circ}$, respectively, are all normal (Saenger, 1988) and almost equal. The orientation of $\mathrm{O}^{\prime}$ with respect to the sugar moiety, described by the $\mathrm{OS}^{\prime}$ $-\mathrm{C}^{\prime}-\mathrm{C}^{\prime}-\mathrm{C}^{\prime}$ torsion angle $\gamma$, is different for molecules $A$ and $B$. In the first we find a $+s c$
Table 2. Bond lengths $(\AA)$, bond angles $\left({ }^{\circ}\right)$ and selected torsion angles $\left({ }^{\circ}\right)$ with e.s.d.'s in parentheses

$\begin{array}{ll}\mathrm{N} 1 A-\mathrm{C} 2 A & 1.397(3) \\ \mathrm{N} 1 A-\mathrm{C} 6 A & 1.373(4) \\ \mathrm{N} 1 A-\mathrm{Cl}^{\prime} A & 1.463(3) \\ \mathrm{C} 2 A-\mathrm{O} 2 A & 1.253(3) \\ \mathrm{C} 2 A-\mathrm{N} 3 A & 1.352(4) \\ \mathrm{N} 3 A-\mathrm{C} 4 A & 1.336(4) \\ \mathrm{C} 4 A-\mathrm{N} 4 A & 1.338(4) \\ \mathrm{C} 4 A-\mathrm{C} 5 A & 1.423(3) \\ \mathrm{C} 5 A-\mathrm{C} 6 A & 1.342(3) \\ \mathrm{Cl}^{\prime} A-\mathrm{C} 2^{\prime} A & 1.509(3) \\ \mathrm{Cl}^{\prime} A-4^{\prime} A & 1.426(3) \\ \mathrm{C}^{\prime} A-\mathrm{C} 3^{\prime} A & 1.501(4) \\ \mathrm{C}^{\prime} A-\mathrm{F}^{\prime} A & 1.413(3) \\ \mathrm{C}^{\prime} A-\mathrm{C}^{\prime} A & 1.520(3) \\ \mathrm{C}^{\prime} A-\mathrm{O}^{\prime} A & 1.447(3) \\ \mathrm{C} 4{ }^{\prime} A-\mathrm{C}^{\prime} A & 1.514(4) \\ \mathrm{C} 5^{\prime} A-\mathrm{O} 5^{\prime} A & 1.418(4)\end{array}$

\begin{tabular}{|c|c|}
\hline $\mathrm{C} 2 A-\mathrm{N} 1 A-\mathrm{C} 6 A$ & $120.7(2)$ \\
\hline $\mathrm{C} 2 A-\mathrm{N} 1 A-\mathrm{Cl}^{\prime} A$ & $118.4(2)$ \\
\hline $\mathrm{C} 6 A-\mathrm{N} 1 A-\mathrm{Cl}^{\prime} A$ & $120 \cdot 9(2)$ \\
\hline $\mathrm{N} 1 A-\mathrm{C} 2 A-\mathrm{O} 2 A$ & $118.9(3)$ \\
\hline $\mathrm{N} 1 A-\mathrm{C} 2 A-\mathrm{N} 3 A$ & $119 \cdot 3(3)$ \\
\hline $\mathrm{O} 2 A-\mathrm{C} 2 A-\mathrm{N} 3 A$ & $121 \cdot 8(3)$ \\
\hline $\mathrm{C} 2 A-\mathrm{N} 3 A-\mathrm{C} 4 A$ & $119.9(2)$ \\
\hline $\mathrm{N} 3 A-\mathrm{C} 4 A-\mathrm{N} 4 A$ & $117 \cdot 3(3)$ \\
\hline $\mathrm{N} 3 A-\mathrm{C} 4 A-\mathrm{C} 5 A$ & $121.8(3)$ \\
\hline $\mathrm{N} 4 A-\mathrm{C} 4 A-\mathrm{C} 5 A$ & $120.9(3)$ \\
\hline $\mathrm{C} 4 A-\mathrm{C} 5 A-\mathrm{C} 6 A$ & $118.0(2)$ \\
\hline $\mathrm{N}: A-\mathrm{C} 6 A-\mathrm{C} 5 A$ & $120 \cdot 3(2)$ \\
\hline $\mathrm{N} 1 A-\mathrm{C}^{\prime} A-\mathrm{C} 2^{\prime} A$ & $114.3(2)$ \\
\hline $\mathrm{N} 1 A-\mathrm{Cl}^{\prime} A-\mathrm{O}^{\prime} A$ & $109 \cdot 1(2)$ \\
\hline $\mathrm{C} 2^{\prime} A-\mathrm{Cl}^{\prime} A-\mathrm{O}^{\prime} A$ & $105 \cdot 1(2)$ \\
\hline $\mathrm{Cl}^{\prime} A-\mathrm{C} 2^{\prime} A-\mathrm{C}^{\prime} A$ & $100 \cdot 9(2)$ \\
\hline $\mathrm{C} 2^{\prime} A-\mathrm{C}^{\prime} A-\mathrm{F}^{\prime} A$ & $107 \cdot 2(2)$ \\
\hline $\mathrm{C} 2^{\prime} A-\mathrm{C} 3^{\prime} A-\mathrm{C} 4^{\prime} A$ & $103.7(2)$ \\
\hline $\mathrm{F} 3^{\prime} A-\mathrm{C} 3^{\prime} A-\mathrm{C} 4^{\prime} A$ & $108.4(2)$ \\
\hline $\mathrm{C} 3^{\prime} A-\mathrm{C} 4^{\prime} . A-\mathrm{O} 4^{\prime} A$ & $106 \cdot 2(2)$ \\
\hline $\mathrm{C}^{\prime} A-\mathrm{C} 4^{\prime} A-\mathrm{C}^{\prime} A$ & $114.9(2)$ \\
\hline $\mathrm{O} 4^{\prime} A-\mathrm{C} 4^{\prime} A-\mathrm{C} 5^{\prime} A$ & $109 \cdot 6(2)$ \\
\hline $\mathrm{Cl}^{\prime} A-\mathrm{O} 4^{\prime} A-\mathrm{C}^{\prime} A$ & $108 \cdot 1(2)$ \\
\hline $\mathrm{C}^{\prime} A-\mathrm{C}^{\prime} A-\mathrm{OS}^{\prime} A$ & $112 \cdot 8(2)$ \\
\hline $\mathrm{C} 2^{\prime} A-\mathrm{Cl}^{\prime} A-\mathrm{O}^{\prime} A-\mathrm{C}^{\prime} A$ & $-28.6(2)$ \\
\hline $\mathrm{O}^{\prime} A-\mathrm{Cl}^{\prime} A-\mathrm{C}^{\prime} A-\mathrm{C}^{\prime} A$ & $39 \cdot 9(2)$ \\
\hline $\mathrm{Cl}^{\prime} A-\mathrm{C} 2^{\prime} A-\mathrm{C} 3^{\prime} A-\mathrm{C} 4^{\prime} A$ & $-35 \cdot 5(2)$ \\
\hline $\mathrm{C} 2^{\prime} A-\mathrm{C} 3^{\prime} A-\mathrm{C}^{\prime} A-\mathrm{O} 4^{\prime} A$ & $19 \cdot 7(2)$ \\
\hline $\mathrm{C}^{\prime} A-\mathrm{C} 4^{\prime} A-\mathrm{O}^{\prime} A-\mathrm{Cl}^{\prime} A$ & $5 \cdot 4(2)$ \\
\hline
\end{tabular}

$\begin{array}{ll}\mathrm{N} 1 B-\mathrm{C} 2 B & 1.407(3) \\ \mathrm{N} 1 B-\mathrm{C} 6 B & 1.364(3) \\ \mathrm{N} 1 B-\mathrm{C} 1^{\prime} B & 1.475(3) \\ \mathrm{C} 2 B-\mathrm{O} 2 B & 1.235(3) \\ \mathrm{C} 2 B-\mathrm{N} 3 B & 1.359(3) \\ \mathrm{N} 3 B-\mathrm{C} 4 B & 1.338(3) \\ \mathrm{C} 4 B-\mathrm{N} 4 B & 1.333(3) \\ \mathrm{C} 4 B-\mathrm{C} 5 B & 1.428(3) \\ \mathrm{C} 5 B-\mathrm{C} 6 B & 1.347(3) \\ \mathrm{C} 1^{\prime} B-\mathrm{C} 2^{\prime} B & 1.519(3) \\ \mathrm{C} 1^{\prime} B-\mathrm{O}^{\prime} B & 1.417(3) \\ \mathrm{C} 2^{\prime} B-\mathrm{C}^{\prime} B & 1.509(3) \\ \mathrm{C} 3^{\prime} B-\mathrm{F}^{\prime} B & 1.408(3) \\ \mathrm{C} 3^{\prime} B-\mathrm{C}^{\prime} B & 1.512(4) \\ \mathrm{C}^{\prime} B-4^{\prime} B & 1.449(4) \\ \mathrm{C} 4^{\prime} B-\mathrm{C}^{\prime} B & 1.514(3) \\ \mathrm{C} 5^{\prime} B-\mathrm{O}^{\prime} B & 1.419(4)\end{array}$

\begin{tabular}{|c|c|}
\hline $\mathrm{C} 2 B-\mathrm{N} 1 B-\mathrm{C} 6 B$ & $120 \cdot 7(2)$ \\
\hline $\mathrm{C} 2 B-\mathrm{N} 1 B-\mathrm{Cl}^{\prime} B$ & $117.5(2)$ \\
\hline $\mathrm{C} 6 B-\mathrm{N} 1 B-\mathrm{Cl}^{\prime} B$ & $121 \cdot 7(2)$ \\
\hline $\mathrm{N} 1 B-\mathrm{C} 2 B-\mathrm{O} 2 B$ & $118 \cdot 3(2)$ \\
\hline $\mathrm{N} 1 B-\mathrm{C} 2 B-\mathrm{N} 3 B$ & $118.8(2)$ \\
\hline $\mathrm{O} 2 B-\mathrm{C} 2 B-\mathrm{N} 3 B$ & $122.9(3)$ \\
\hline $\mathrm{C} 2 B-\mathrm{N} 3 B-\mathrm{C} 4 B$ & $120 \cdot 0(2)$ \\
\hline $\mathrm{N} 3 B-\mathrm{C} 4 B-\mathrm{N} 4 B$ & $118 \cdot 3(2)$ \\
\hline $\mathrm{N} 3 B-\mathrm{C} 4 B-\mathrm{C} 5 B$ & $121 \cdot 8(2)$ \\
\hline $\mathrm{N} 4 B-\mathrm{C} 4 B-\mathrm{C} 5 B$ & $120 \cdot 0(2)$ \\
\hline $\mathrm{C} 4 B-\mathrm{C} 5 B-\mathrm{C} 6 B$ & $117.6(3)$ \\
\hline $\mathrm{N} 1 B-\mathrm{C} 6 B-\mathrm{C} 5 B$ & $120.8(3)$ \\
\hline $\mathrm{N} 1 B-\mathrm{Cl}^{\prime} B-\mathrm{C}^{\prime} B$ & $113 \cdot 3(1)$ \\
\hline $\mathrm{N} 1 B-\mathrm{Cl}^{\prime} B-\mathrm{O}^{\prime} B$ & $108 \cdot 2(2)$ \\
\hline $\mathrm{C} 2^{\prime} B-\mathrm{Cl}^{\prime} B-\mathrm{O}^{\prime} B$ & $106.0(2)$ \\
\hline $\mathrm{Cl}^{\prime} B-\mathrm{C} 2^{\prime} B-\mathrm{C} 3^{\prime} B$ & $101 \cdot 7(2)$ \\
\hline $\mathrm{C} 2^{\prime} B-\mathrm{C} 3^{\prime} B-\mathrm{F}^{\prime} B$ & $109 \cdot 0(2)$ \\
\hline$C 2^{\prime} B-C 3^{\prime} B-C 4^{\prime} B$ & $103 \cdot 5(2)$ \\
\hline $\mathrm{F}^{\prime} B-\mathrm{C} 3^{\prime} B-\mathrm{C} 4^{\prime} B$ & $109 \cdot 6(2)$ \\
\hline$C 3^{\prime} B-C 4^{\prime} B-O 4^{\prime} B$ & $106.4(2)$ \\
\hline${ }^{\prime} 3^{\prime} B-\mathrm{C}^{\prime} B-\mathrm{C}^{\prime} B$ & $113.4(2)$ \\
\hline${ }^{\prime} 4^{\prime} B-C 4^{\prime} B-C 5^{\prime} B$ & $108 \cdot 4(3)$ \\
\hline $\mathrm{Cl}^{\prime} B-\mathrm{O}^{\prime} B-\mathrm{C}^{\prime} B$ & $109 \cdot 4(2)$ \\
\hline $\mathrm{C} 4^{\prime} B-\mathrm{C}^{\prime} B-\mathrm{O}^{\prime} B$ & $106 \cdot 3(2)$ \\
\hline $\mathrm{C} 2^{\prime} B-\mathrm{C} 1^{\prime} B-\mathrm{O} 4^{\prime} B-C 4^{\prime} B$ & $-20 \cdot 4(2)$ \\
\hline $\mathrm{O} 4^{\prime} B-\mathrm{Cl}^{\prime} B-\mathrm{C} 2^{\prime} B-\mathrm{C}^{\prime} B$ & $34 \cdot 2(2)$ \\
\hline $\mathrm{Cl}^{\prime} B-\mathrm{C} 2^{\prime} B-\mathrm{C} 3^{\prime} B-\mathrm{C} 4^{\prime} B$ & $-34 \cdot 3(2)$ \\
\hline$C 2^{\prime} B-C 3^{\prime} B-C 4^{\prime} B-O 4^{\prime} B$ & $23.4(2)$ \\
\hline $\mathrm{C}^{\prime} B-\mathrm{C} 4^{\prime} B-\mathrm{O} 4^{\prime} B-\mathrm{Cl}^{\prime} B$ & $-1.9(2)$ \\
\hline
\end{tabular}

Table 3. Geometry of intra- and intermolecular hydrogen bonds $\left(\AA,{ }^{\circ}\right)$ with e.s.d.'s in parentheses

$\begin{array}{lccc}X-\mathrm{H} \cdots Y & d(\mathrm{H} \cdots Y) & d(X \cdots Y) & X-\mathrm{H} \cdots Y \\ \mathrm{O} 5^{\prime} A-\mathrm{H} 1 A \cdots \mathrm{O} 2 A^{\mathrm{i}} & 2 \cdot 06(3) & 2 \cdot 825(3) & 162(3) \\ \mathrm{N} 4 A-\mathrm{H} 11 A \cdots \mathrm{O} 2 A^{\mathrm{ii}} & 2 \cdot 25(3) & 3 \cdot 012(3) & 153(3) \\ \mathrm{N} 4 A-\mathrm{H} 12 A \cdots \mathrm{N} 3 B^{\mathrm{iii}} & 2 \cdot 09(3) & 3.029(3) & 172(3) \\ \mathrm{N} 4 B-\mathrm{H} 11 B \cdots \mathrm{O} 2 B^{\text {iv }} & 2 \cdot 12(4) & 2 \cdot 961(3) & 164(3) \\ \mathrm{O} 5^{\prime} B-\mathrm{H} 1 B \cdots 5^{\prime} A^{\mathrm{iv}} & 2 \cdot 06(3) & 2 \cdot 843(3) & 162(3) \\ \mathrm{N} 4 B-\mathrm{H} 12 B \cdots \mathrm{N} 3 A^{\mathrm{v}} & 2 \cdot 09(3) & 3 \cdot 002(3) & 172(3)\end{array}$

Symmetry code: (i) $x, y-1, z$; (ii) $x+1, y, z$; (iii) $x+1, y+2, z-1$; (iv) $x-1, y, z ;$ (v) $x-1, y-2, z+1$.

orientation $\left[\gamma=63.4(4)^{\circ}\right]$, while in molecule $B[\gamma=$ $-71.4(4)^{\circ}$ ], we find the unusual $-s c$ conformation.

The packing of the crystal is totally determined by a network of hydrogen bonds, as shown in Fig. 2 and summarized in Table 3. Base pairing between molecules $A$ and $B$ occurs, since every $\mathrm{H} 12$ is hydrogen bonded to $\mathrm{N} 3$ of the opposite molecule. In this way, a pseudo ring between the bases of $A$ and $B$ is formed. The centre of this pseudo ring coincides with a pseudo-inversion centre between both bases, which explains the pseudocentrosymmetric nature of the crystal. A strong 


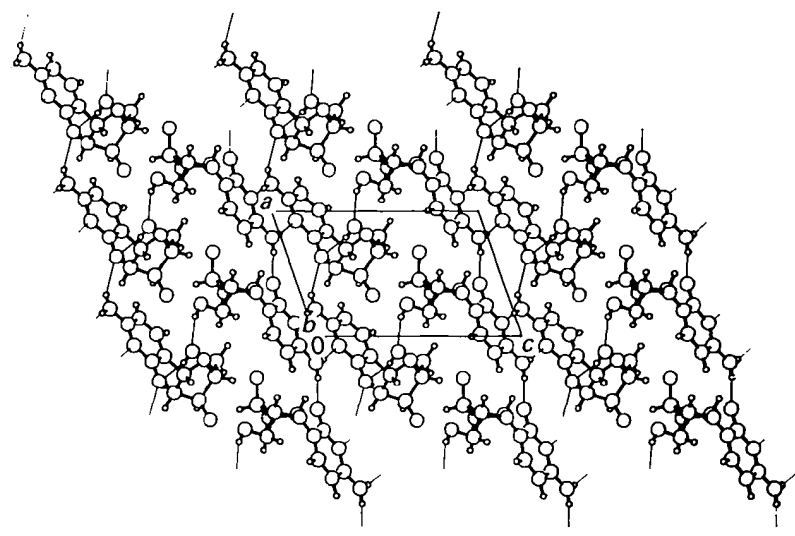

Fig. 2. PLUTO plot (Motherwell \& Clegg, 1978) of the crystal packing along $\mathbf{b}$. Thin lines indicate hydrogen bonds.

hydrogen-bond network along the crystallographic $a$ axis is formed by $\mathrm{O} 2 B$ and $\mathrm{H} 11 B-\mathrm{N} 4 B$, while the packing along $\mathbf{b}$ is determined by hydrogen bonds between $\mathrm{O}^{\prime} A-\mathrm{H} 1 A$ and $\mathrm{O} 2 A$.

The authors wish to thank J. P. Van Cuyck for his help in preparing the figures.

\section{References}

Allen, F. H., Kennard, O., Watson, D. G., Brammer, L., Orpen, A. G. \& Taylor, R. (1987). J. Chem. Soc. Perkin Trans. 2, pp. S1-S19.
Beurskens, P. T., Beurskens, G., Strumpel, M. \& Nordman, C. E. (1987). Patterson and Pattersons, edited by J. P. Glusker, B. K. Patterson \& M. Rossi, pp. 356-367. Oxford Univ. Press.

Beurskens, P. T., Bosman, W. P., Doesburg, H. M., van den Hark, T. E. M., Prick, P. A. J., Noordik, J. H., Beurskens, G., Gould, R. O. \& Parthasarathi, V. (1983). DIRDIF. Applications of Direct Methods to Difference Structure Factors. Univ. of Nijmegen, The Netherlands.

Enraf-Nonius (1985). Structure Determination Package. EnrafNonius, Delft, The Netherlands.

Everaert, D. H., Peeters, O. M., Blaton, N. M., De ranter, C. J., Van Aerschot, A. \& HerdewiJn, P. (1991). Acta Cryst. C47, 678-680.

Herdewijn, P., Balzarini, J., De Clerce, E., Pauwels, R., Baba, M., Broder, S. \& Vanderhaeghe, H. (1987). J. Med. Chem. 30, 1270-1278.

Ibers, J. A. \& Hamilton, W. C. (1964). Acta Cryst. 17, 781-782.

IUPAC-IUB JoINT COMMISSION ON BIOCHEMICAL NOMENCLATURE (1983). Pure Appl. Chem. 55, 1273-1280.

Lalitha, H. N., Ramakumar, S. \& Viswamitra, M. A. (1989). Acta Cryst. C45, 1652-1655.

Main, P., Fiske, S., Hull, S. E., Lessinger, L., Germain, G., DeClerCQ, J.-P. \& Woolfson, M. M. (1982). MULTAN82. A System of Computer Programs for the Automatic Solution of Crystal Structures from X-ray Diffraction Data. Univs. of York, England, and Louvain, Belgium.

Motherwell, W. D. S. \& ClegG, W. (1978). PLUTO. Program for plotting molecular and crystal structures. Univ. of Cambridge, England.

Nardelli, M. (1983). Comput. Chem. 7, 95-98.

Nyburg, S. C. (1974). Acta Cryst. B30, 253-254.

SaEnger, W. (1988). Principles of Nucleic Acid Structure. New York: Springer Verlag.

Stewart, R. F., Davidson, E. R. \& Simpson, W. T. (1965). J. Chem. Phys. 42, 3175-3187.

Stoe \& Co. (1985). REDU4. Data Reduction Program. Stoe \& Co., Darmstadt, Germany.

Acta Cryst. (1991). C47, 835-837

\title{
Structure of 1-(2-Deoxy- $\beta$-D-ribopyranosyl)-5-iodouracil*
}

\author{
By H. L. De Winter, N. M. Blaton, O. M. Peeters and C. J. De Ranter $\dagger$ \\ Laboratorium voor Analytische Chemie en Medicinale Fysicochemie, \\ Instituut voor Farmaceutische Wetenschappen, Katholieke Universiteit Leuven, Van Evenstraat 4, \\ B-3000 Leuven, Belgium
}

ANd A. Van Aerschot and P. Herdewijn

Laboratorium voor Farmaceutische Chemie, Rega Institute for Medical Research, Katholieke Universiteit Leuven, B-3000 Leuven, Belgium

(Received 30 April 1990; accepted 17 September 1990)

\begin{abstract}
Deoxy- $\beta$-D-ribopyranosyl)-5-iodouracil, $\mathrm{C}_{9} \mathrm{H}_{11} \mathrm{IN}_{2} \mathrm{O}_{5}, M_{r}=354 \cdot 10$, monoclinic, $P 2_{\mathrm{I}}, a$

\footnotetext{
* Structural Studies on Modified Nucleosides. Part VII. Part VI: De Winter, Blaton, Peeters, De Ranter, Van Aerschot \& Herdewijn (1991).

$\dagger$ To whom correspondence should be addressed.
}

$0108-2701 / 91 / 040835-03 \$ 03.00$
$=5.458(3), \quad b=8.237(4), \quad c=12.812(6) \AA, \quad \beta=$ $98.42(4)^{\circ}, V=569.8(5) \AA^{3}, Z=2, D_{m}=2.05, D_{x}=$ $2.063 \mathrm{Mg} \mathrm{m}^{-3}, \quad \lambda($ Mo $K \alpha)=0.71069 \AA, \quad \mu=$ $2.789 \mathrm{~mm}^{-1}, \quad F(000)=344, \quad T=293 \mathrm{~K}$, final $R=$ 0.039 for 1701 unique observed $[F \geq 4 \sigma(F)]$ reflections. The sugar ring adopts a slightly flattened chair conformation. The heterocyclic base is placed in an (C) 1991 International Union of Crystallography 